\title{
Is ERCC1 a reliable prognostic protein biomarker in non-small-cell lung cancer?
}

After resection for non-small-cell lung cancer (NSCLC) cisplatin-based postoperative chemotherapy may be administered. How do we predict which patients will benefit from cisplatin chemotherapy?

This group previously published that the excision repair cross-complementation group 1 (ERCC1) protein acts as a prognostic biomarker, predicting the benefit of cisplatin-based chemotherapy after NSCLC resection. ERCC1 plays a crucial role in repairing DNA structures, damaged by cisplatin-based chemotherapy. Using immunohistochemical analysis, patients with NSCLC and no detectable ERCC1 had longer disease-free survival after cisplatin-based chemotherapy than those positive for ERCC1.

Debate over the specificity of the antibody 8F1, used to stain for ERCC1, led this group to evaluate its use. They showed that $8 \mathrm{~F} 1$ had lost its predictive value in determining the response of NSCLC to cisplatin-based chemotherapy. When 8F1 was used to re-stain tissue sections in which it had previously had a predictive value, its ability was lost. The authors' postulate, that this loss of predictive value could be due to a new batch of $8 \mathrm{~F} 1$ antibody.

Sixteen antibodies for ERCC1 were assayed by immunoblot to determine their ability to detect specific isoforms of ERCC1. None of these antibodies could determine just one isoform of ERCC1. The expression of non-functional ERCC1 isoforms may lead to discrepant results. The authors show that of the four isoforms of ERCC1, only ERCC1-202 was active. Strong homology between the four isoforms means developing a ERCC1-202-specific antibody would be difficult.

This paper highlights the technical issues that may interfere when using an immunohistochemical signal as a predictive biomarker. The need to assess multiple isoforms is paramount, before a biomarker is used to determine clinical practice.

Questions

- Do any other commercially available antibodies stain for ERCC1 in immunohistochemistry analysis?

- Have you checked the validity of the antibody 8F1 in other tumours such as cholangiocarcinoma, gastric cancer etc?

- What is your next step-it seems that producing an isoform-specific ERCC1-202 antibody is impossible? Can we rely on mRNA detection? What 'functional' analysis of ERCC1 could you do in tissue samples?

Hannah J Durrington

Correspondence to Dr Hannah J Durrington, NIHR Clinical Lecturer in Respiratory Medicine, University of Manchester, AV Hill Building, Oxford Road, Manchester M13 9PT, UK; hannahd@doctors.org.uk

Competing interests None.

Provenance and peer review Not commissioned; internally peer reviewed.

To cite Durrington HJ. Thorax 2014;69:345.

Published Online First 10 July 2013

Thorax 2014;69:345. doi:10.1136/thoraxjnl-2013-204086

REFERENCE

1 Friboulet L, Olaussen KA, Pignon J-P, et al. ERCC1 isoform expression and DNA repair in non-small-cell lung cancer. $N$ Engl J Med 2013;368:1101-10. 